\title{
Design of Control System for Tracked Tank Cleaning Robot
}

\author{
Wei Dongpo ${ }^{1,2, a}$, Meng Fanzhao ${ }^{1,2, ~ b, ~ * ~}$ \\ ${ }^{1}$ School of Mechanical Engineering, Shandong Huayu University of Technology, Dezhou 253034, China \\ ${ }^{2}$ Dezhou Municipal Key Laboratory of Industrial Robot Control, Dezhou 253034, China \\ a wei31028@yeah.net, bmfzhthy@163.com \\ *corresponding author
}

Keywords: Robots; Control system; Hydraulic system; PLC

\begin{abstract}
A hydraulic control system for tank cleaning robot based on PLC was designed to afford the operating safety and flexibility. Firstly all component elements of the hydraulic control system were confirmed after analyzing the structural characteristics and functions of the robots. Secondly, on the basis of working principle and process of every component element, a hydraulic system circuit was designed for the robot and the achieving process of every component element was analyzed. Last, the PLC control system were respectively devised.
\end{abstract}

\section{Introduction}

At present, domestic petrochemical enterprises usually use manual methods to clean oil storage tanks [1]. In such a flammable, explosive and toxic harsh environment, relying on manual use of simple tools to clean the equipment, there are problems such as poor safety, low cleaning efficiency, long construction period, and environmental pollution. The chemical tank cleaning method was adding chemical reagents to the sludge at the bottom of the tank, heating the mixture, chemically reacting the chemical with components such as sludge, and then withdrawing the mixture out of the tank. Chemical methods also have certain limitations. Many chemical cleaning agents have a certain corrosive effect on metal materials such as tank wall panels and pipeline accessories, which affects the service life of the tank to a certain extent. At the same time, the chemical cleaning method was difficult to completely remove some abnormally hard knots with a wall thickness of $100 \mathrm{~mm}$, and it needed to be manually removed. At this time, the toxic gas in the storage tank was also easy to cause casualties. The treatment and discharge of chemical cleaning waste liquids were also difficult to recycle and easily cause chemical pollution. In addition, the oil content of oil tank sludge was about $60 \%$ to $70 \%$. If it was not treated directly, it will waste resources and cause serious pollution to the surrounding soil, groundwater and the environment.

Tracked Robot was designed for tank sludge cleaning, which can move freely inside the tank, flushing and scraping the tank, and instead of manually cleaning the tank. A hydraulic control system for tank cleaning robot based on PLC was designed to afford the operating safety and flexibility [2].

\section{System composition}

The hydraulic control system of the tank cleaning robot includes a hydraulic system and a control system. The function of the hydraulic system was to convert the mechanical energy of the engine with oil as the medium, convert the oil pump into hydraulic energy, transfer it to the cylinder, hydraulic motor, etc. into mechanical energy, and then drive the actuators to realize various movements and working processes. The hydraulic system includes actuators, control elements, and the like. The hydraulic actuators of the robot mainly include a hydraulic cylinder capable of linear reciprocating motion and a hydraulic motor capable of reciprocating rotary motion. The actuator system was composed as shown in Figure 1, including the mobile unit and the pusher unit. The mobile unit was composed of four symmetrically arranged track wheels driven by a hydraulic motor, 
which can drive the track to make the robot realize all-round movement in the plane. The pusher unit was composed of shovel and two hydraulic cylinders that control the lifting of the shovel, and with the high-pressure jet nozzle on the robot, the pusher operation was performed [3].

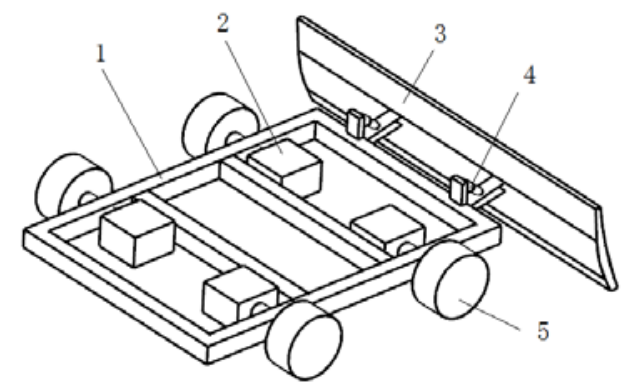

\section{1 chassis}

2 hydraulic motor

3 shovel

4 hydraulic cylinder

5 track wheel

Figure 1 Hydraulic actuator system

The hydraulic system controls the movement of the actuator through various control elements such as the valve body [4-5]. The robot hydraulic system control element controls the operating state of the hydraulic circuit. The relief valve, accumulator and pressure monitor decompress and stabilize the circuit for safety protection; The three-position four-way electromagnetic reversing valve and the speed regulating valve control the extension, retraction, rotation direction and rotation speed of the hydraulic cylinder and the hydraulic motor. According to the function and structural characteristics, the tank cleaning robot used the PLC control system to control each actuator.

\section{Hydraulic system design}

According to the working principle and process of the above-described robot moving unit and the pushing unit, the hydraulic system was designed as shown in Figure 2.

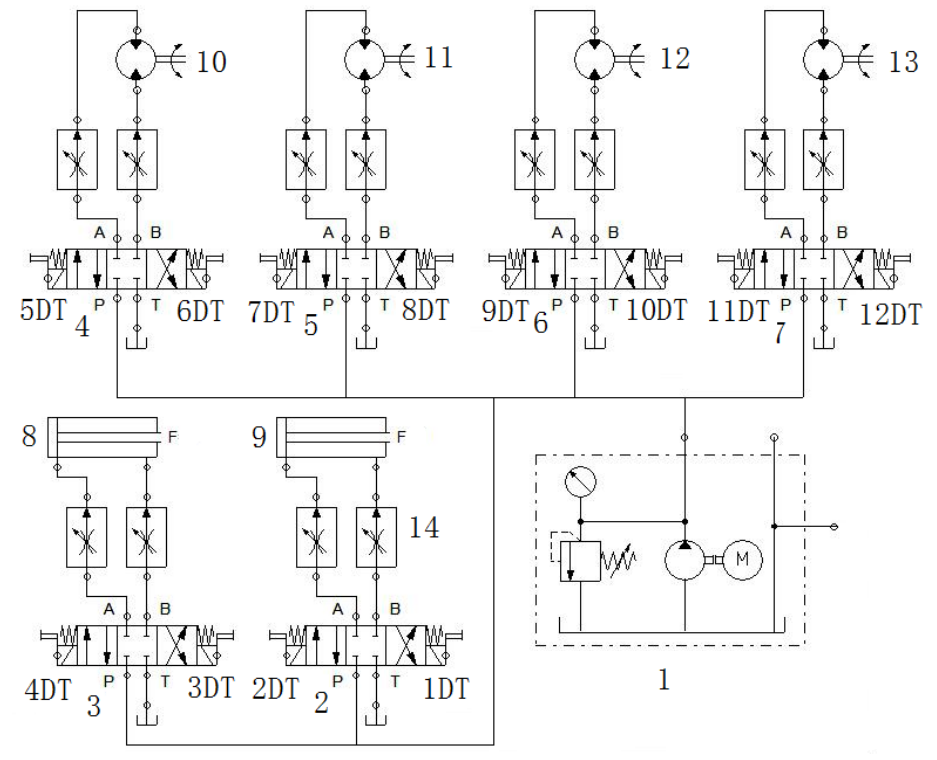

1 hydraulic station

2-7 three-position four-way electromagnetic reversing valve 8-9 hydraulic cylinder 10-11 right track wheel hydraulic motor 12-13 left track wheel hydraulic motor 14 speed control valve

Figure 2 Hydraulic system schematic

\subsection{Moving}

According to the characteristics of the track mechanism, when the robot needed to move, it was only necessary to coordinately control the rotation of the four hydraulic motors 10-13 to realize the in-situ steering and the linear movement.

(a) Turning in place. When the 5DT, 7DT, 10DT and 12DT or 6DT, 8DT, 9DT and 11DT were energized, the right 2 track wheels rotated clockwise, and the left 2 track wheels rotated counter clockwise or the right 2 track wheels rotated counter clockwise, and the left 2 track wheels rotated clockwise at the same speed, and the robot turned to the left or right.

(b) Linear movement. When the system pressure was constant and the 5DT, 7DT, 9DT and 
11DT or 6DT, 8DT, 10DT and 12DT of the Three-position four-way electromagnetic reversing valve 2-7 were energized, the 4 track wheels rotated clockwise or counter clockwise at the same speed, the robot Move straight forward or backward. In either case, the robot rotates or moves along an irregular curve.

\subsection{Pushing}

The shovel was controlled by two hydraulic cylinders. When the 1DT and 3DT were energized, the piston rods of the hydraulic cylinders 8 and 9 were retracted, and the shovel was lifted; when the 2DT and 4DT were energized, the piston rods of the hydraulic cylinders 8 and 9 were extended, pushed the shovel down and pushing sludge.

\section{PLC control system design}

The operation of each of the above hydraulic cylinders and hydraulic motors was controlled by the three-position four-way solenoid valve. In order to realized PLC control of the loop, the PLC's input/output signals were used to control the state of the 1DT-12DT to achieve the sequence of actions [6]. According to the robot's working process, 16 digital input points and 14 digital output points were required. The system used SIMATIC S7-200 series CPU226 DC/DC/DC type PLC [7]. According to the function analysis of the hydraulic system, some input/output point assignments and functions of the PLC can be designed as shown in Table 1-2. The I/O terminal wiring diagram of the PLC was shown in Figure 3.

Table 1 Input signal I/O allocation and function comparison table

\begin{tabular}{ccc}
\hline Function & Name & Add. \\
start up button & IB1 & X0 \\
stop button & IB2 & X1 \\
Hydraulic cylinder 1 extended & IQ1 & X2 \\
Hydraulic cylinder 1 retracted & IQ2 & X3 \\
Hydraulic cylinder 2 extends & IQ3 & X4 \\
Hydraulic cylinder 2 retracted & IQ4 & X5 \\
Right front hydraulic motor forward & IQ5 & X6 \\
Right front hydraulic motor reverse & IQ6 & X7 \\
Right rear hydraulic motor forward & IQ7 & X8 \\
Right rear hydraulic motor reverse & IQ8 & X9 \\
Left front hydraulic motor forward & IQ9 & X10 \\
Left front hydraulic motor reverse & IQ10 & X11 \\
Left rear hydraulic motor forward & IQ11 & X12 \\
Left rear hydraulic motor reverse & IQ12 & X13 \\
Filter alarm & ID1 & X14 \\
alarm reset & ID2 & X15 \\
\hline
\end{tabular}

Table 2 Output signal I/O allocation and function comparison table

\begin{tabular}{ccc}
\hline Function & Name & Add. \\
\hline start up button & OB1 & Y0 \\
stop button & OB2 & Y1 \\
Hydraulic cylinder 1 extended & DT1 & Y2 \\
Hydraulic cylinder 1 retracted & DT2 & Y3 \\
Hydraulic cylinder 2 extends & DT3 & Y4 \\
Hydraulic cylinder 2 retracted & DT4 & Y5 \\
Right front hydraulic motor forward & DT5 & Y6 \\
Right front hydraulic motor reverse & DT6 & Y7 \\
Right rear hydraulic motor forward & DT7 & Y8 \\
Right rear hydraulic motor reverse & DT8 & Y9 \\
Left front hydraulic motor forward & DT9 & Y10 \\
Left front hydraulic motor reverse & DT10 & Y11 \\
Left rear hydraulic motor forward & DT11 & Y12 \\
Left rear hydraulic motor reverse & DT12 & Y13 \\
\hline
\end{tabular}




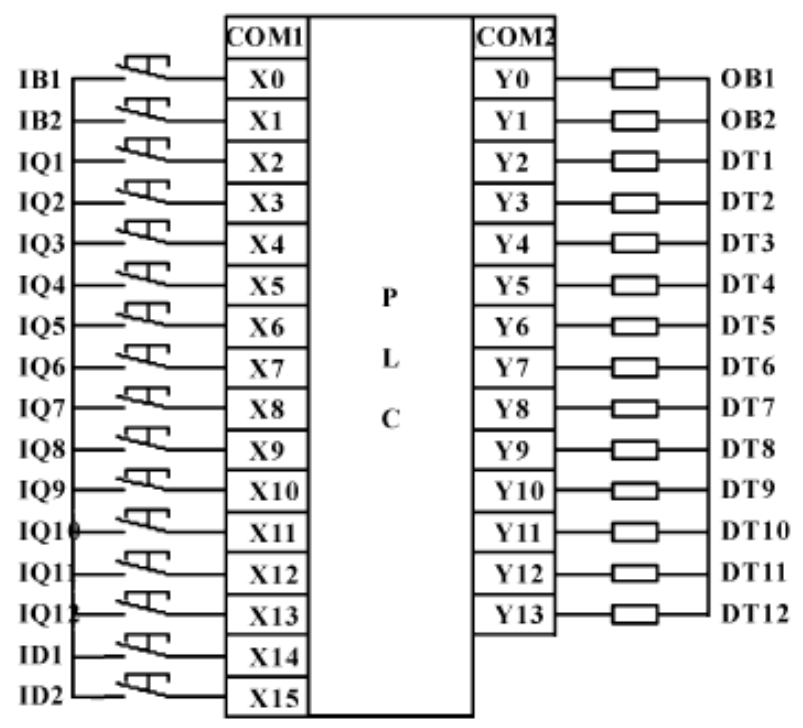

Figure 3 PLC I/O terminal wiring diagram

\section{Conclusion}

In this paper, through the research of robot control technology, a hydraulic system based on PLC control technology was designed for the tracked tank cleaning robot with visual operation. The system has the characteristics of operating safety and flexibility. It can accurately control the forward, backward, steering and using shovel cleaning of the robot, and at the same time, clean the sludge in the oil tank with the suction pump.

\section{Acknowledgements}

This work was supported by the Project of Shandong province higher education science and technology program (NO. J17KA034).

\section{References}

[1] Li Dongsheng, Sun Xiaoyan, Yang Hailong, Zhou Hongjun. (2011) Review of cleaning technique for crude oil storage tank. Cleaning World, 6: 13-16.

[2] Xie Yuanyuan, Wang Lei. (2018) Research on Control System of Multi-DOF Robot with Hydraulic Transmission. Hydraulics Pneumatics \& Seals, 04: 42-45.

[3] Meng Fanzhao, Chen Fude, Tan Dening. (2018) Analysis on steering mechanics model of tracked tank cleaning robot. Advances in engineering research, 152, 81-85.

[4] Zhang R, Li S, Jin Y, et al. (2001) Competitive Surface Interactions of Critical Additives Interacted with Piston Ring/Cylinder Liner Components under Lubricated Running-in Conditions. Science in China, Ser A, 44: 149-155.

[5] Jiang Bo, Liu Qingyun. (2011) Design and Simulation of a Hydraulic Finger Mechanism Based on Actuated Joints. Mechanical Science and Technology for Aerospace Engineering, 30(8): 1402-1408.

[6] Zhu Linghong. (2011) The Movement Design of Hydraulic Manipulator Based on PLC. Machine Tool \& Hydraulics, 39(6): 79-104.

[7] Liu Meijun. (2017) Siemens PLC programming and application. Bei Jing: China Machine Press, 28-29. 